\title{
An investigation of copper chlorophyllin solution for low-cost optical devices calibration in chlorophyll measurement
}

\author{
Bayu Taruna Widjaja Putra ${ }^{1, *}$, Risky Setiawan Purwoko ${ }^{1}$, Indarto Indarto ${ }^{1}$, and Peeyush Soni ${ }^{2}$ \\ ${ }^{1}$ Laboratory of Precision Agriculture Geo-Informatics. Faculty of Agricultural Technology, Jember University, Jember 68121, \\ East Java, Indonesia \\ ${ }^{2}$ Department of Agricultural and Food Engineering, Indian Institute of Technology Kharagpur: Kharagpur, West Bengal, India
}

Received: 6 July 2019 / Accepted: 19 August 2019

\begin{abstract}
Chlorophylls and turbidity are related parameters deemed important in predicting water quality, especially in the freshwater fisheries sector. In other hand, chlorophyll contents in vegetation are often used as a measure for estimating plant health. To ensure the chlorophyll content, appropriate analysis techniques are needed, which include, yet not limited to, dimethylsulfoxide (DMSO), acetone extraction analysis, and measuring turbidity using expensive equipment. Measurements using these methods are still not reliable, especially for smallholders. In addition, calibration of a digital camera for these purposes requires several efforts of preparing a series of chemical analyses and the qualities of newly developed cameras have increased. Thus, calibration needs to be taken into account for improving the accuracy in estimating chlorophyll contents. This study posits fundamental findings germane to the potential use of chlorophyllin for calibrating the optimal instrument, like consumer-grade cameras. Sodium copper chlorophyllin is commonly used as food additive and ingredients, and medical treatments. However, we can gain another benefit of chlorophyllin, particularly for developing low-cost optical instruments for estimating chlorophyll content through hydro-color-based with promising accuracy.
\end{abstract}

Keywords: Chlorophyllin / absorbance / sensing system / chlorophyll / turbidity / camera

\section{Background}

Chlorophyll is an important component of photosynthesis available in plants and green biotic (entity) in water. Chlorophylls are the pigments which are related to photosynthesis and found in plants and green biotic entity which trap the sunlight. High content of chlorophylls in plants indicates healthy condition. However, excessive chlorophyll in water can lead to decreased ecosystem health and reduced fish productivities. Thus, with these properties, chlorophyll can be an effective indicator in decision making concerned with monitoring plant health, preventing water pollution, and examining nutrient availability for fish.

Smallholder farmers and small-scale freshwater fish farming still grapple with the difficulty in measuring water quality, particularly due to the high cost of sensors and equipment. Some farmers even do not have sufficient technology. As shown by field sampling, the current problems include increased cost of chemical laboratory analysis and unreliable analysis result. Common method of laboratory analysis is chlorophyll extraction using acetone and dimethylsulfoxide (DMSO). These methods are widely applied yet time-consuming. Moreover, the samples cannot be used any

\footnotetext{
* Corresponding author: bayu@unej.ac.id
}

further after being measured using the methods aforementioned. The solution obtained from chlorophyll extraction using DMSO or acetone can be stored only for a short-term basis. Thus, it is difficult to obtain the same specification of chlorophyll assay for future analysis. Several studies show different procedures in chlorophyll extraction using acetone and DMSO. Several methods claim that the use of $80 \%$ acetone and absorbance spectral read at 645 and $665 \mathrm{~nm}$ [1], as well as 646 and $663 \mathrm{~nm}$ [2], can accurately measure chlorophyll content [2]. Another study also proposes using $100 \%$ acetone and extracting the absorbance value read at 661.6 and $664.5 \mathrm{~nm}$ for measuring chlorophyll-a and chlorophyll-b. In addition, numerous spectral indices using reflectance method are also proposed to estimate chlorophyll content [3-5]. Several non-destructive methods using cameras/sensors are proposed for estimating chlorophyll $[6,7]$ and turbidity $[8,9]$. Due to the rapid advancement of technology, the cameras available in the market have always been improved with enhanced specification. As such, using a single standardized camera on a long-term basis is hardly feasible. Therefore, standardization is only possible for every model of camera. Another issue is related to the short storage period of water solutions, therefore making future accurate re-measurement unfeasible due to unstable chemical composition. 


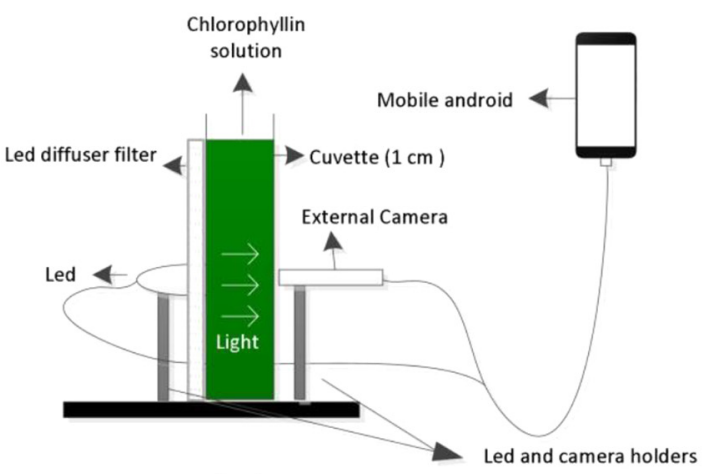

(a)



(c)

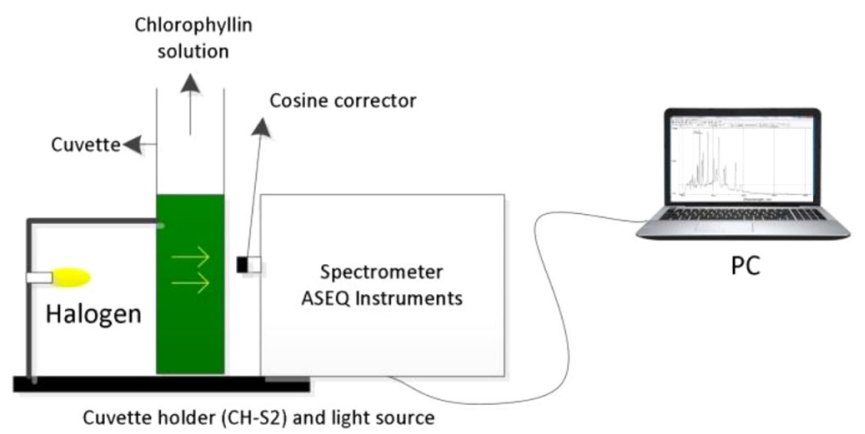

(b)

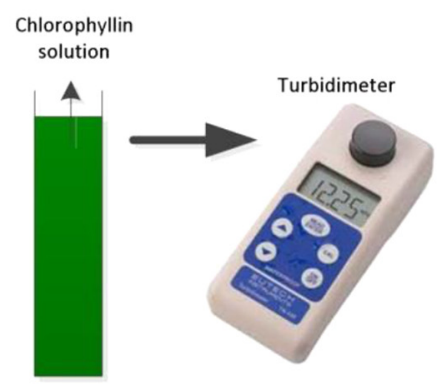

(d)

Fig. 1. Different methods of hydro color measurement using (a) low-cost external camera with a custom android application; (b) spectrometer; (c) iPhone/Samsung camera; (d) turbidimeter.

To obtain standardized value and measured object, chlorophyllin sodium copper salt, often called chlorophyllin, is used in this study. Several types of chlorophyllin [10] have been produced, namely chlorophyllin $\mathrm{A}$ and $\mathrm{B}$, with the molecular formula in the acid form of $\mathrm{C}_{34} \mathrm{H}_{34} \mathrm{~N}_{4} \mathrm{O}_{5}$ and $\mathrm{C}_{34} \mathrm{H}_{32} \mathrm{~N}_{4} \mathrm{O}_{6}$, respectively. while chlorophyll a [11] and b [12] have molecular formula of $\mathrm{C}_{55} \mathrm{H}_{72} \mathrm{MgN}_{4} \mathrm{O}_{5}$ and $\mathrm{C}_{55} \mathrm{H}_{70} \mathrm{MgN}_{4} \mathrm{O}_{6}$ respectively. There are similarities between chlorophyll and chlorophyllin, one of which is the fact that chlorophyllin a and b hold five-O and six-O atoms, respectively. Few companies like Alfa Aesar and Thermo Fisher Scientific produce these kinds of chlorophyllin in standard value and assay. Cooper chlorophyllin is a chlorophyll-derived food colorant, commercially available as a complex mixture of different chlorin molecules [13]. Since the chlorophyllin is soluble in water, adding acetone and centrifuges processes is no longer necessary for chlorophyll extraction in chlorophyllin. These steps can reduce the time of calibration process. We assume that measured chlorophyll values obtained from chemical analysis using DMSO or acetone extraction can be reflected to chlorophyllin in particular amount. Thus, chlorophyll a and b contents in chlorophyllin can represent the amount of chlorophylls in water solutions.

This study offers a potential method in estimating chlorophylls and turbidity using digital camera and spectrometer by analyzing the amount of copper chlorophyllin solution obtained from absorbance reading. Issues concerning the use of chlorophyllin for sensor calibration and hydro color application have not been underresearched. Thus, the aims of this study are (a) evaluating the use of copper chlorophyllin as a rapid and low-cost substance for calibrating the optical instrument for estimating chlorophyll content based on hydro color characteristics; (b) evaluating the existing methods in determining chlorophyll content; and (c) evaluating chlorophyllin solution through turbidimeter.

\section{Material and methods}

\subsection{Materials and samples preparation}

Chlorophyllin - coppered trisodium salt (Alfa aesar, UK) was used in this study. This chlorophyllin formula is $\mathrm{C}_{34} \mathrm{H}_{31} \mathrm{CuN}_{4} \mathrm{Na}_{3} \mathrm{O}_{6}$ with assay (unspecified): $95 \%$ (dry wt. basis) and $\mathrm{Cu}$ element is about 3.5-6.5\%. In the preparation, there were 150 samples packed in zipper plastics, each of which contained $2 \mathrm{mg}$ chlorophyllin. Afterward, each pack of $2 \mathrm{mg}$ chlorophyllin was mixed up in a beaker glass. The glasses containing chlorophyllin were filled with distilled water, at different amount. Each of these samples was then measured twice and averaged.

For measurement, three digital cameras were used in this study. The first camera, which was a custom camera, was an external camera with a light source powered by the camera battery and was integrated with the Android-based application (Fig. 1a). The second and third cameras were the internal cameras obtained from iPhone and Samsung S5, respectively 
Table 1. Proposed indices for estimating water quality through absorbance techniques.

\begin{tabular}{lllll}
\hline No & Indices & Formula & Allotment & Reference \\
\hline 1 & Chlorophyll-a & $12.7\left(A_{663}\right)-2.69\left(A_{645}\right)$ & Spectrometer & {$[1,2]$} \\
& & $11.24\left(A_{661.6}\right)-2.04\left(A_{664.8}\right)$ & & \\
2 & Chlorophyll-b & $22.9\left(A_{645}\right)-4.68\left(A_{663}\right)$ & Spectrometer & {$[1,2]$} \\
3 & Normalized R & $20.13\left(A_{664.8}\right)-5.03\left(\mathrm{~A}_{661.6}\right)$ & & \\
4 & Normalized G & $\frac{R}{R+G+B}$ & Camera & {$[14]$} \\
5 & Normalized B & $\frac{G}{R+G+B}$ & Camera & {$[14]$} \\
6 & hue & $\frac{B}{R+G+B}$ & Camera & {$[14]$} \\
7 & Simple ratio green/red & $120+\frac{60(B-R)}{[\max (R, G, B)-\min (R, G, B)\}}$ & Camera & {$[14]$} \\
8 & Simple ratio blue/green & $\mathrm{G} / \mathrm{R} / \mathrm{G}$ & Camera & {$[14]$} \\
\hline
\end{tabular}

Note: $1 \mathrm{~nm}$ steps were used in this study; $A_{661.6}$ and $A_{664.8}$ were altered to $A_{662}$ and $A_{665}$ respectively.

(Fig. 1c). Both cameras used the same light source as that used on the first one. These cameras were used for collecting data concerning absorbance spectra within particular distance and illumination. Each sample was captured individually using these cameras. Spectral absorbance of each sample was also measured using ASEQ LR1-T with spectral resolution of $0.2 \mathrm{~nm}$ and spectral range from $400 \mathrm{~nm}$ to $1000 \mathrm{~nm}$ (ASEQ Instruments, Canada) (Fig. 1b). Turbidity was measured using a turbidimeter (Waterproof Portable TN100, Eutech Instruments Pte Ltd, Singapore) (Fig. 1d).

\subsection{Indices}

Two methods involving the absorbance of spectrometer were applied to measure chlorophyll. Several indices were proposed for the camera and analyzed using custom android-application. The indices are shown in Table 1.

\section{Results and discussion}

\subsection{Spectral performance in different chlorophyllin contents}

Spectral performance and captured images of different chlorophyllin concentration are shown in Figure 2. This initial analysis shows that absorbance characteristics from different chlorophyllin concentration change gradually. This is indicated by the color changes captured by the consumergrade camera and the measurement results obtained from turbidimeter. Both parameters indicate similar results with respect to the changes in spectral characteristics. similar to the study conducted by [16], which posits that the absorption of chlorophyll is strong in areas within $640-670 \mathrm{~nm}$ and 410-470 nm. Thus, the use of consumer-grade camera and turbidimeter has a potential relationship with spectral absorbance. Further analysis needs to be carried out in order to reveal the relationship among spectrometer, camera, and turbidimeter to analyze the characteristics of chlorophyll content of a, b and total chlorophyll.

\subsection{Chlorophyll-a, chlorophyll-b, and total chlorophylls}

Two methods for determining the amount of chlorophyll a and b proposed by $[1,2]$ were evaluated in this step. Both

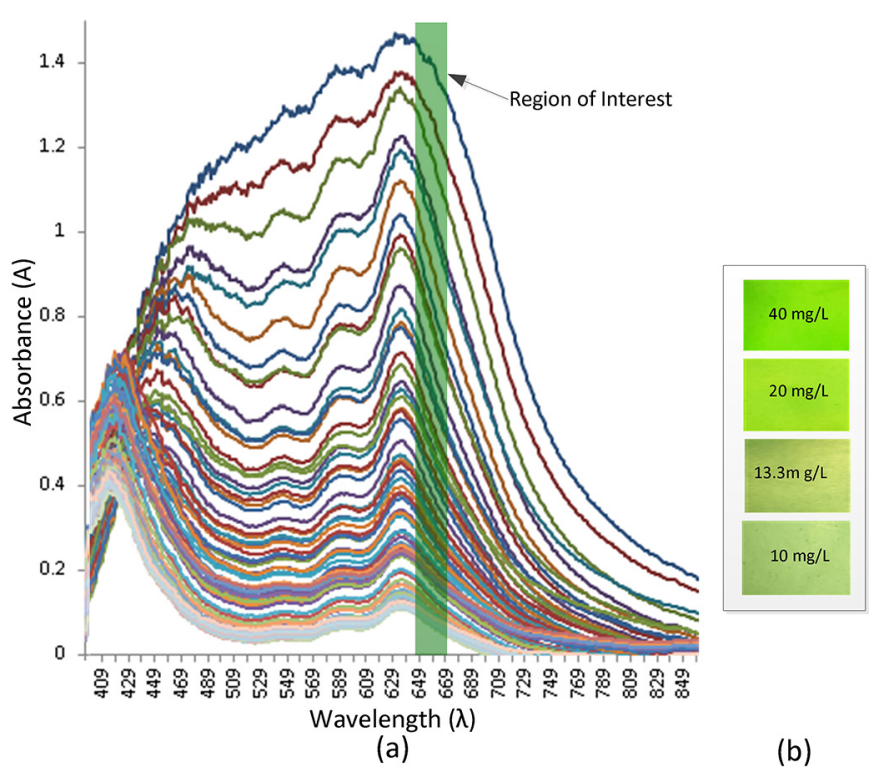

Fig. 2. (a) Spectral information of different chlorophyllin concentrations and their turbidity. (b) Camera-generated visualizations of different chlorophyllin concentrations.

methods reveal strong relationship between chlorophyllin concentrations and determination of chlorophyll $\mathrm{a}$ and $\mathrm{b}$ in chlorophyllin (Fig. 3). However, in these solutions, the concentration of chlorophyll-b was higher than that of chlorophyll-a. This is evidenced by the use of sodium copper chlorophyllin with a formula of $\mathrm{C}_{34} \mathrm{H}_{31} \mathrm{CuN}_{4} \mathrm{Na}_{3} \mathrm{O}_{6}$; this type of chlorophyllin is similar to chlorophyll-b in that both bind six O-atoms in their formula $[10,12]$.

The next steps investigate the most suitable method in determining the chlorophyll content. In this study, two methods for determining the total chlorophyll solution were compared. Figure 4a shows the relationship between chlorophyllin content and total chlorophyll. The total chlorophyll content measured using Arnon method indicated higher value than that measured using Lichtenthaler method. However, it still does not show the relationship between chlorophyll concentrations in chlorophyllin. 



Fig. 3. Relationship of different types of chlorophyll (a and b) in chlorophyllin using different methods of chlorophyll determination; Lichtenthaler (left) and Arnon (right).

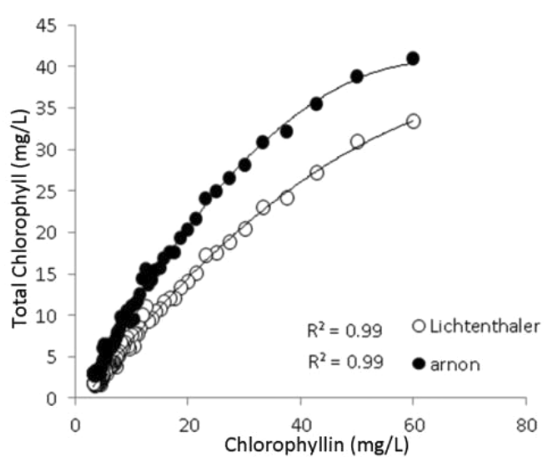

(a)

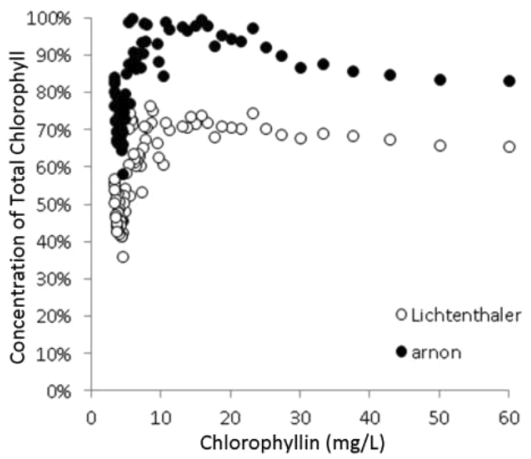

(b)

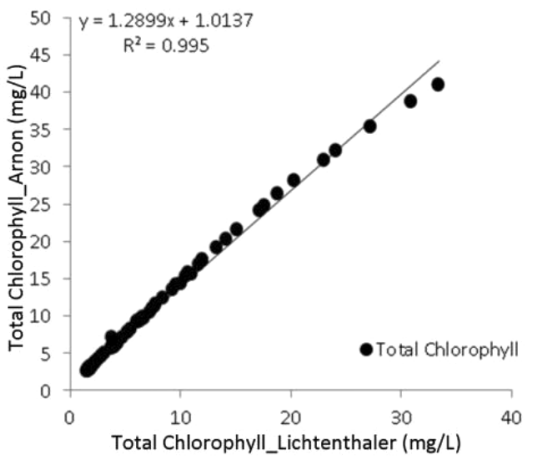

(c)

Fig. 4. (a) Correlation between total chlorophyll and chlorophyllin concentrations in $\mathrm{mg} / \mathrm{L}$; (b) relationship between total chlorophyll concentration (assay in percent \%) and chlorophyllin concentration $(\mathrm{mg} / \mathrm{L})$ with different methods of chlorophyll determination; (c) correlation between Lichtenthaler and Arnon method in determining total chlorophyll.

The relationship between total chlorophyll concentration and chlorophyllin content is shown in Figure 4b. Determination of chlorophyll content using the Arnon method is more accurate and meets the product specifications stipulated by Alfa Aesar with concentration close to $100 \%$. By contrast, Lichtentaler method can only estimate up to $75 \%$ of the total chlorophyll concentration.

Although the results obtained using Lichtenthaler method is lower than Arnon method, these methods provide strong relationship (Fig. 4c).

\subsection{Turbidity level of chlorophyllin}

According to our findings, turbidimeter can be used to estimate chlorophyll content, as long as the solution is soluble in water or leaves no sediments. As such, if sediments are evident, it is necessary to perform centrifugation process at the outset of analysis. However, This chlorophyllin is one of the highly stable and water-soluble derivatives of chlorophyll which are formed by replacing the central magnesium atom by copper atom [17]. The relationship between turbid values obtained using turbidimeter and total chlorophyll measured using different determination methods is presented in Figure 5.

\subsection{Chlorophyll and turbidity estimation using cameras}

In this study, proposed indices were evaluated for estimating chlorophyll content using a digital camera (Fig. 6). According to our findings, the $\mathrm{B} / \mathrm{G}$ index performs better than other proposed indices. This is corroborated by the study conducted by $[15,18]$, which point out that B and $\mathrm{G}$ bands are suitable for estimating the chlorophyll content. Specific models for estimating chlorophyll a or chlorophyll b cannot be used for estimating chlorophyll contents in water or plants, due to the ratio between these chlorophylls in water or plant is not the same. Research findings obtained by [19-21] show that the assay of 


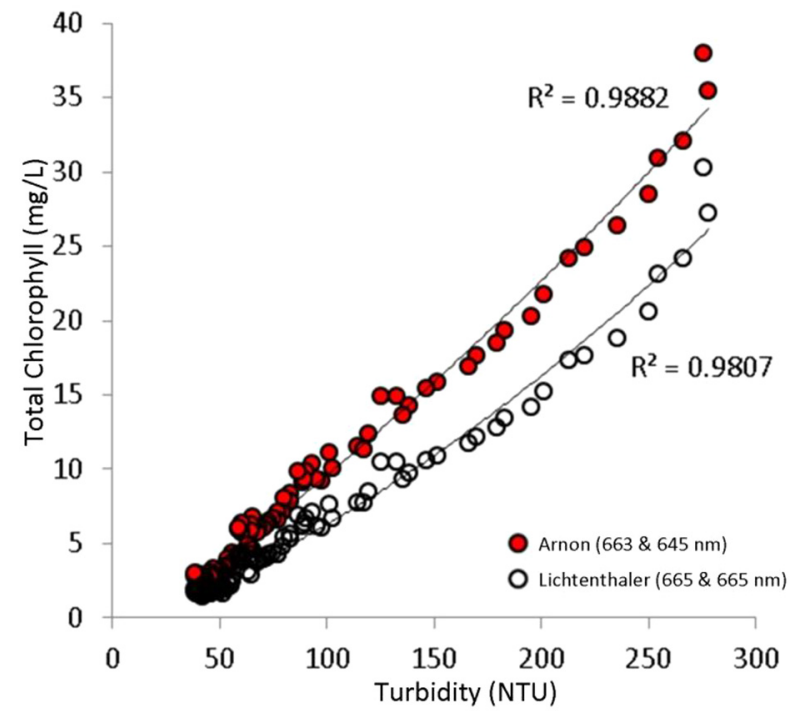

Fig. 5. Correlation between total chlorophyll in chlorophyllin solution and turbidity levels.

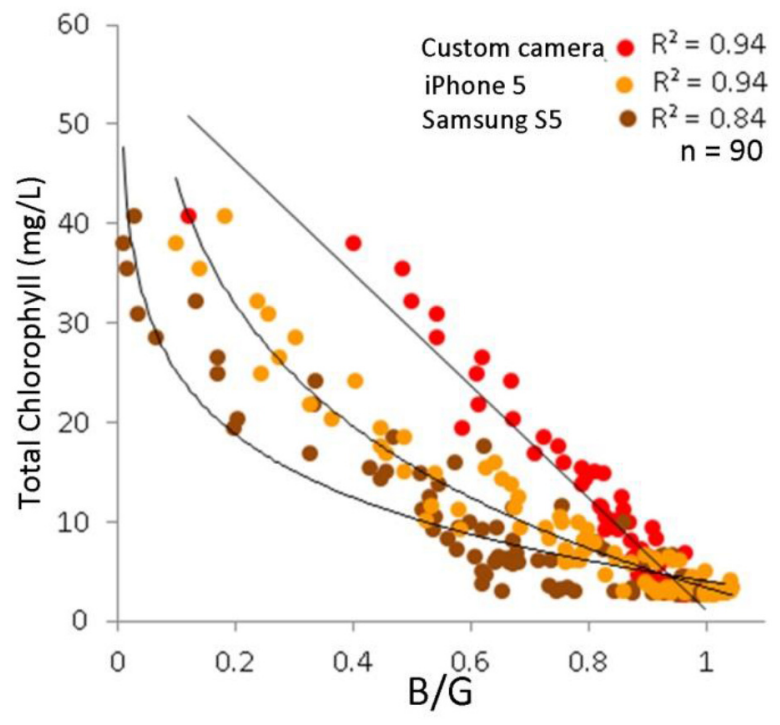

Fig. 7. Relationship between total chlorophyll and B/G index obtained from different cameras.
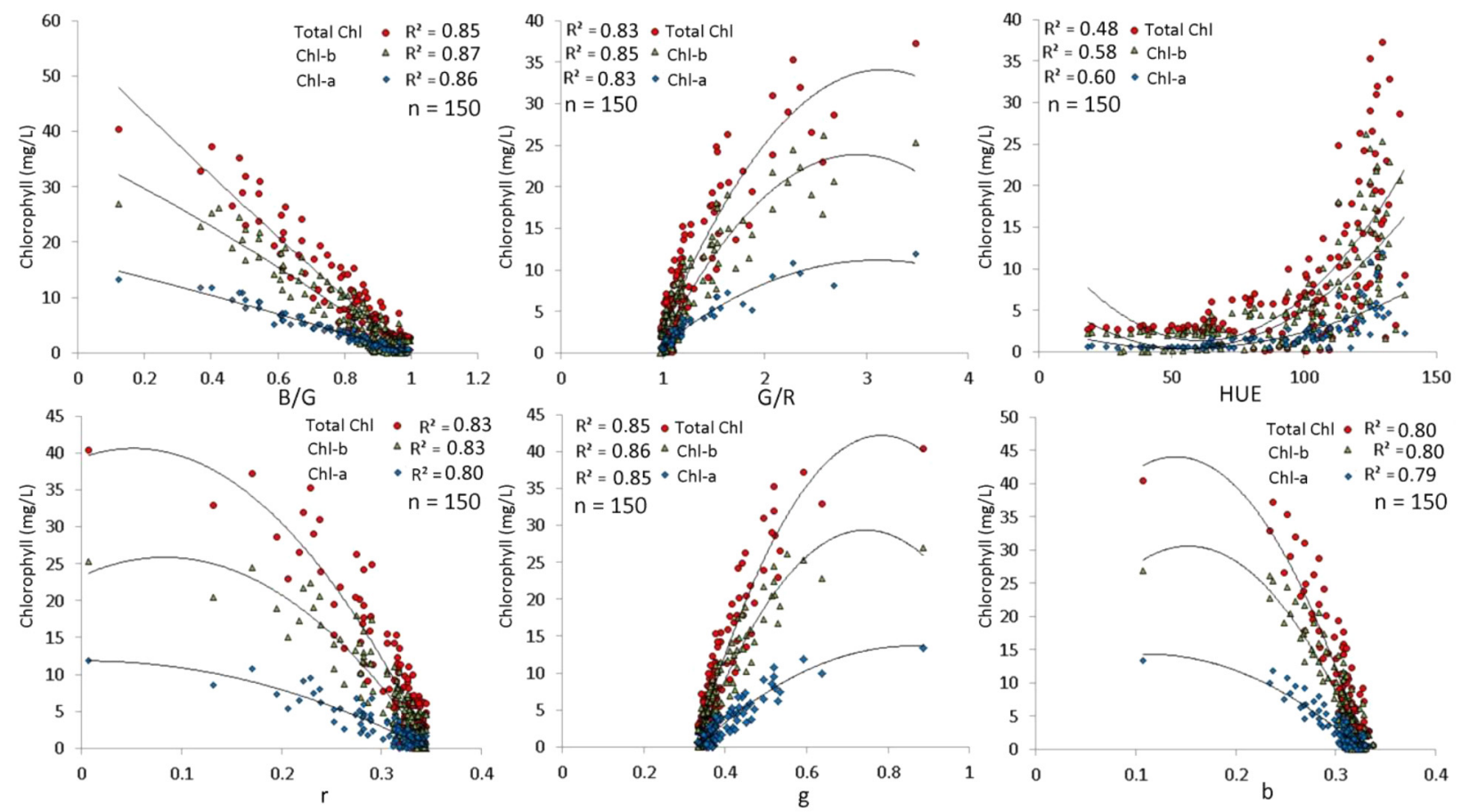

Fig. 6. Relationship between chlorophyll contents in chlorophyllin and indices.

chlorophyll-a in living objects (i.e. leaves and algae) provides more than chlorophyll-b. However, a general recommendation in estimating total chlorophyll can be made through these methods.

In addition, three digital cameras, namely custom camera, iPhone, and Samsung S5 were tested and compared in estimating total chlorophyll content and turbidity levels of chlorophyllin-based water solution using $\mathrm{B} / \mathrm{G}$ index. Figure 7 shows that three digital cameras are strongly correlated with total chlorophyll and turbidity level. However, if high estimation accuracy is the main concern, low-cost camera is recommended for smallholders.

\section{Conclusion}

The use of copper chlorophyllin is a low-cost method for calibrating purposes and can be used as a standard solution for optical calibration and evaluation without any field 
experiment using plant tissue or algae in particular amount. The experimental results lead to the following conclusions:

- copper chlorophyllin can be a reference for conducting rapid and repeatable optical calibration and use as an alternative method for calibrating hydro color from different optical devices like consumer-grade digital cameras;

- the use of consumer-grade camera which incorporates B and $\mathrm{G}$ band proves strong relationship in estimating chlorophyll contents and its turbidity level; it also can be used as a general recommendation in estimating total chlorophyll content;

- the wavelength of $662 \mathrm{~nm}$ and $665 \mathrm{~nm}$ is used to determine chlorophyll content by calibrating it to the common chlorophyll determination proposed by Arnon;

- a turbidity value is strongly related to the total chlorophyll. Thus, turbidity can be used/operationalized to estimate total chlorophyll;

- determination of chlorophyll a and b still requires further research, due to different ratio between chlorophyll a and $\mathrm{b}$ in each vegetation or algae.

This project was funded by Kurita-AIT Research Grant 2017. The authors would like also to acknowledge support received from Laboratory of Precision Agriculture and Geo-informatics University of Jember - Indonesia.

\section{References}

1. D.I. Arnon, Copper enzymes in isolated chloroplasts. Polyphenoloxidase in Beta vulgaris, Plant Physiol. 24, 1-15 (1949)

2. H.K. Lichtenthaler, Chlorophylls and carotenoids: pigments of photosynthetic biomembranes, Methods Enzymol. 148, 350-382 (1987)

3. A. Gitelson, The peak near $700 \mathrm{~nm}$ on radiance spectra of algae and water: relationships of its magnitude and position with chlorophyll concentration, Int. J. Remote Sens. 13, 3367-3373 (1992)

4. E.J. D'Sa, R.L. Miller, Bio-optical properties in waters influenced by the Mississippi River during low flow conditions, Remote Sens. Environ. 84, 538-549 (2003)

5. H.B. Jiao, Y. Zha, J. Gao, Y.M. Li, Y.C. Wei, J.Z. Huang, Estimation of chlorophyll- $a$ concentration in Lake Tai, China using in situ hyperspectral data, Int. J. Remote Sens. 27, 4267-4276 (2006)

6. T. Leeuw, E. Boss, The HydroColor app: above water measurements of remote sensing reflectance and turbidity using a smartphone camera, Sensors 18, 256 (2018)
7. T. Guimarães, M. Veronez, E. Koste, L. Gonzaga, F. Bordin, L. Inocencio, A. Larocca, M. de Oliveira, D. Vitti, F. Mauad, An alternative method of spatial autocorrelation for chlorophyll detection in water bodies using remote sensing, Sustainability 9, 416 (2017)

8. T. Toivanen, S. Koponen, V. Kotovirta, M. Molinier, P. Chengyuan, Water quality analysis using an inexpensive device and a mobile phone, Environ. Syst. Res. 2, 9 (2013)

9. L. Parra, J. Rocher, J. Escrivá, J. Lloret, Design and development of low cost smart turbidity sensor for water quality monitoring in fish farms, Aquacult. Eng. 81, 10-18 (2018)

10. Sodium copper chlorophyllin | C34H31CuN4Na3O6 PubChem, https://pubchem.ncbi.nlm.nih.gov/compound/ 23725082\#section=Top

11. Chlorophyll a | C55H72MgN4O5 - PubChem, https:// pubchem.ncbi.nlm.nih.gov/compound/chlorophyll_a\#sec tion $=$ Top

12. Chlorophyll B | C55H70MgN4O6 - PubChem, https:// pubchem.ncbi.nlm.nih.gov/compound/chlorophyll_b

13. T. Tumolo, U.M. Lanfer-Marquez, Copper chlorophyllin: a food colorant with bioactive properties? Food Res. Int. 46, 451-459 (2012)

14. B.T.W. Putra, P. Soni, Enhanced broadband greenness in assessing Chlorophyll a and b, Carotenoid, and Nitrogen in Robusta coffee plantations using a digital camera, Precis. Agric. 19, 238-256 (2018)

15. L.M. Goddijn, M. White, Using a digital camera for water quality measurements in Galway Bay, Estuar. Coast Shelf Sci. 66, 429-436 (2006)

16. C. Chen, N. Gong, F. Qu, Y. Gao, W. Fang, C. Sun, Z. Men, Effects of carotenoids on the absorption and fluorescence spectral properties and fluorescence quenching of chlorophyll a, Spectrochim. Acta A 204, 440-445 (2018)

17. A.A.M. Farag, Optical absorption of sodium copper chlorophyllin thin films in UV-vis-NIR region, Spectrochim. Acta A 65, 667-672 (2006)

18. S. Damirchi, A.-K.K. Maliheh, T. Heidari, Z. Es'haghi, M. Chamsaz, A comparison between digital camera and spectrophotometer for sensitive and selective kinetic determination of brilliant green in wastewaters, Spectrochim. Acta A 206, 232-239 (2019)

19. A.T. Netto, E. Campostrini, J.G. de Oliveira, R.E. Bressan-Smith, Photosynthetic pigments, nitrogen, chlorophyll a fluorescence and SPAD-502 readings in coffee leaves, Sci. Hortic. (Amsterdam). 104, 199-209 (2005)

20. C.S. Daughtry, C. Walthall, M. Kim, E.B. de Colstoun, J. McMurtrey, Estimating corn leaf chlorophyll concentration from leaf and canopy reflectance, Remote Sens. Environ. 74, 229-239 (2000)

21. S.W. Thorne, E.H. Newcomb, C.B. Osmond, Identification of chlorophyll $\mathrm{b}$ in extracts of prokaryotic algae by fluorescence spectroscopy, Proc. Natl. Acad. Sci. USA 74, 575-578 (1977)

Cite this article as: Bayu Taruna Widjaja Putra, Risky Setiawan Purwoko, Indarto Indarto, Peeyush Soni, An investigation of copper chlorophyllin solution for low-cost optical devices calibration in chlorophyll measurement, Int. J. Metrol. Qual. Eng. 10, $10(2019)$ 